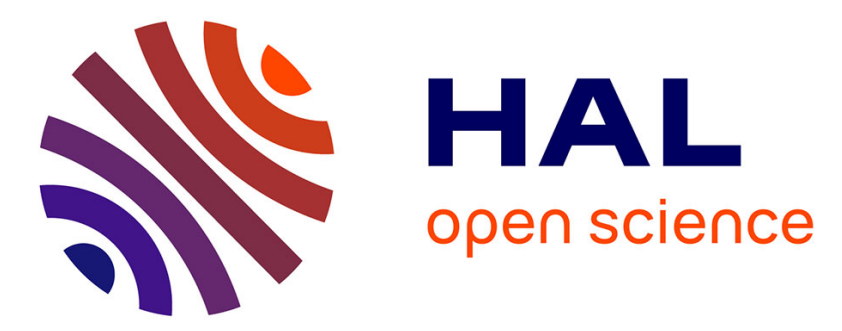

\title{
Prediction of a body's structural impedance and scattering properties using correlation of random noise
}

Sandrine Rakotonarivo, W. A. Kuperman, Earl G. Williams

\section{To cite this version:}

Sandrine Rakotonarivo, W. A. Kuperman, Earl G. Williams. Prediction of a body's structural impedance and scattering properties using correlation of random noise. Journal of the Acoustical Society of America, 2013, 134, pp.4401-4411. 10.1121/1.4828833] . hal-01128144

\section{HAL Id: hal-01128144 \\ https://hal.science/hal-01128144}

Submitted on 29 Mar 2016

HAL is a multi-disciplinary open access archive for the deposit and dissemination of scientific research documents, whether they are published or not. The documents may come from teaching and research institutions in France or abroad, or from public or private research centers.
L'archive ouverte pluridisciplinaire HAL, est destinée au dépôt et à la diffusion de documents scientifiques de niveau recherche, publiés ou non, émanant des établissements d'enseignement et de recherche français ou étrangers, des laboratoires publics ou privés. 


\title{
Prediction of a body's structural impedance and scattering properties using correlation of random noise
}

\author{
Sandrine T. Rakotonarivo a) and W. A. Kuperman \\ Scripps Institution of Oceanography, University of California-San Diego, 9500 Gilman Drive, La Jolla, \\ California 92093-0238
}

\author{
Earl G. Williams \\ Naval Research Laboratory, Code 7106, Washington, DC 20375
}

(Received 18 March 2013; revised 14 October 2013; accepted 22 October 2013)

\begin{abstract}
This paper derives a method to estimate the structural or surface impedance matrix (or equivalently the inverse of the structural Green's function) for an elastic body by placing it in an encompassing and spatially random noise field and cross-correlating pressure and normal velocity measurements taken on its surface. A numerical experiment is presented that utilizes a cross-correlation method to determine the structural impedance matrix for an infinite cylindrical shell excited by a spatially random noise field. It is shown that the correlation method produces the exact analytic form of the structural impedance matrix. Furthermore, using standard impedance formulations of the scattered and incident pressure fields at the object surface that are based on the equivalent source method and using this estimated structural impedance, a prediction of the scattered acoustic field at any position outside of the object can be made for any given incident field. An example is presented for a point (line) source near a cylindrical shell and when compared with the analytical result, excellent agreement is found between the scattered fields at a radius close to the shell.
\end{abstract}

(C) 2013 Acoustical Society of America. [http://dx.doi.org/10.1121/1.4828833]

PACS number(s): 43.40.Fz, 43.20.Gp, 43.40.Qi [RKS]

Pages: $4401-4411$

\section{INTRODUCTION}

The structural Green's function describes the in vacuo response of a structure to excitation forces applied to its surface. This Green's function or its inverse, the structural impedance, can be used to predict the scattered field from the object when placed in a fluid under any incident field conditions. In this case, the loading effect of the fluid on the structure must be known and is modeled by the acoustic impedance and the internal impedance. ${ }^{1}$ Those two quantities depend only upon the shape of the object's surface and the outer medium properties, and thus are independent of the object's structural parameters (see Fig. 1).

The acoustic and internal impedances can be deduced using the Helmholtz integral equation (HIE),${ }^{1-3}$ replacing the object with a surface integral, or by the equivalent source method (ESM), replacing the object with a surface populated with point monopole sources. ${ }^{4-6}$ No matter what the method, numerically deriving the scattering properties of a complex object with a large number of internal degrees of freedom is ultimately a very costly endeavor (see Zampolli et al. paper ${ }^{7}$ and references therein). The structural Green's function or structural impedance must be modeled either with an analytical formulation for structures of simple shape or otherwise with finite element methods. ${ }^{4}$ Alternatively, measurements of the scattered field with a more or less complete set of incident fields and a dense set of field points in an effort to deduce the structural impedance are also performed by a very involved procedure. ${ }^{8}$

\footnotetext{
a) Author to whom correspondence should be addressed. Electronic mail: srakotonarivo@ucsd.edu
}

The analytic relationship between the structural impedance (compliance) of a shell/plate-like object and the scattered field is clearly outlined by Borgiotti. ${ }^{9}$ He uses impedance concepts along with a discretization of the Helmholtz integral equation to formulate a scattering formalism. In the paper by Gaumond, ${ }^{8}$ a method for estimating the structural impedance matrix is introduced that estimates the surface forces by using the measured far-field scattered pressure field and the known incident field for cylindrical and spherical geometries through the use of far-field $T$ matrix operators. However, this method is not capable of capturing the structural near-field $\left(\lambda_{s}<\lambda\right.$ where $\lambda_{s}$ and $\lambda$ are the structural and acoustic wavelengths, respectively) that often rapidly decays away from the surface. The near-field is necessary to predict the scattering from forces on or near the surface. Recently, a significant series of papers by Bobrovnitskii ${ }^{110,11}$ has formulated an impedance theory of sound scattering and further makes use of the structural (surface) impedance matrix to optimally design surface coatings for the reduction of scattered field signatures.

In this paper, we derive a method to obtain the structural or surface impedance matrix by placing the object in an encompassing noise field and cross correlating pressure and normal velocity measurements taken on its surface. Using the standard definition of the structural impedance matrix, we show that it can be robustly acquired by the correlation method presented in Sec. II. In Sec. III, we present a numerical example of the correlation method to determine the structural impedance matrix for an infinite cylindrical shell. In Sec. III C, the structural impedance matrix is derived by a numerical experiment using a finite-element multi-physics calculation (COMsol Multiphysics) to simulate the surface pressure and normal velocity from a dense field of uncorrelated noise 


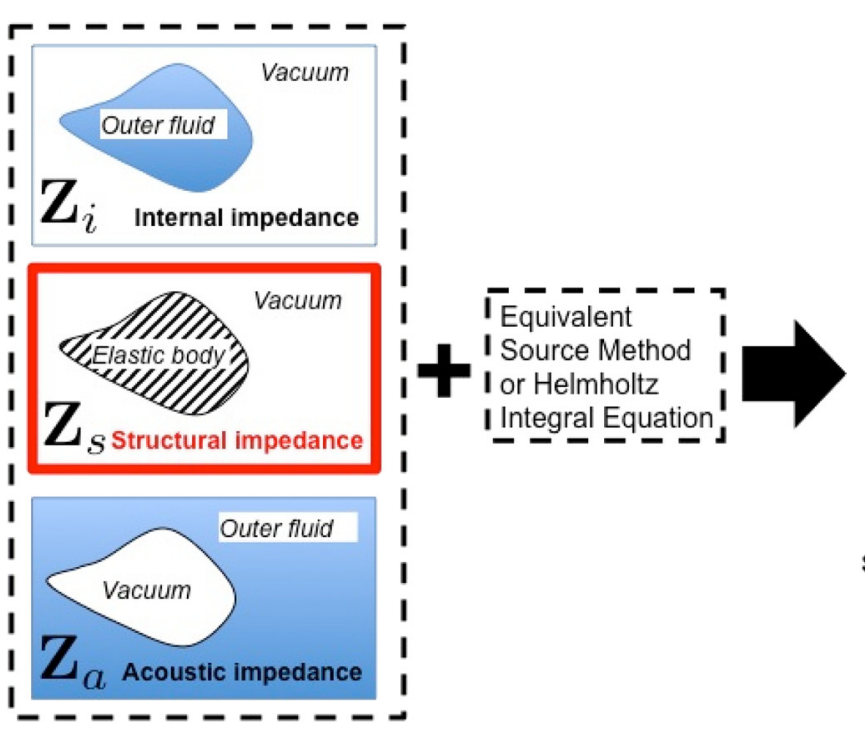

sources placed at all angles in the far-field. Cross correlations are performed on this simulated data, and the structural impedance matrix is computed. Compared to the exact solution, the structural impedance matrix computed by cross correlation agrees very well. In Sec. IV, we review the impedance formulations (i.e., formulations with acoustic and internal impedances) that rely upon the determination of the structural impedance to predict the scattered acoustic fields given only the incident field. The equivalent source method is used to derive the impedances of the incident and scattered fields at the surface of the scatterer. Finally, using the ESM and the structural impedance derived from the numerical experiment presented in Sec. III, the scattered field from a point source near the shell is predicted at a radius close to the shell. Estimation of the scattered field shows excellent agreement with the exact result.

\section{ESTIMATING THE STRUCTURAL IMPEDANCE BY CROSS CORRELATION}

Consider a linearly elastic object with surface $\Omega$ excited into vibration at a frequency $\omega$ by a distribution of forces on its surface $f(\mathbf{x})$ defined as positive in the outward direction. We limit our discussions to harmonic fields and the time dependence $e^{-i \omega t}$ is suppressed in our analysis. The outward-positive normal component of the vibration velocity at the surface is defined by $v(\mathbf{y})$ where $\mathbf{x}$ and $\mathbf{y}$ are vectors in coordinate space from a common origin to $\Omega$. The structural Green's function $G_{s}(\mathbf{y}, \mathbf{x})$ provides an integral relationship between $v(\mathbf{y})$ and $f(\mathbf{x})$ (Refs. 12-14) on the surface of the object when it is placed in vacuo,

$$
v(\mathbf{y})=\int_{\Omega} G_{s}(\mathbf{y}, \mathbf{x}) f(\mathbf{x}) d^{2} \mathbf{x} .
$$

The integral equation is discretized to form a vector/matrix equation and then inverted to give

$$
\mathbf{Z}_{s} \mathbf{v}=\mathbf{f}
$$

where $\mathbf{v}$ and $\mathbf{f}$ (force per unit area) are column vectors of length $N$ spanning the complete surface $\Omega$ and $\mathbf{Z}_{s}$ is an $N \times N$ matrix representing a discretized inverse of the structural
FIG. 1. (Color online) Three impedances are required to describe the scattering problem using either the equivalent source method or the Helmholtz integral equation: $\mathbf{Z}_{a}, \mathbf{Z}_{i}$, and $\mathbf{Z}_{s}$, which are the acoustic, the internal, and the structural impedances, respectively. The acoustic and the internal impedances characterize the vibration of the outer fluid outstide and within the body surface and thus only depend on the body shape and the outer fluid property. The structural impedance characterizes the response of the elastic body in vacuum, thus only depends on the object's structural parameters. This paper focuses on deriving a new method to estimate this structural impedance.
Green's function. $\mathbf{Z}_{s}$ is called the structural impedance matrix and represents the impedance of the object in vacuum. The impedance matrix $\mathbf{Z}_{s}$ depends uniquely upon the elastic properties and geometry of the object but not the external fluid properties. It is sometimes called the dry impedance matrix, ${ }^{9}$ the stiffness matrix, ${ }^{4}$ or the surface impedance matrix. ${ }^{1}$ The forces can arise from excitation of the body due to acoustic sources in the volume outside the object as well as reaction forces due to mass loading from fluid on the exterior of the surface. If the forces arise from acoustic sources and mass loading, a pressure $\mathbf{p}$, a column vector spanning the complete surface, is generated on the surface (defined as positive when the medium is in compression), and equation Eq. (2) still describes the dynamics,

$$
\sum_{j=1}^{N} z_{l j} v_{j}=-p_{l}
$$

where the elements $z_{l j}$ of the structural impedance matrix $\mathbf{Z}_{s}$ connect the pressure, $p_{l}$, at the $l$ th surface node with the normal velocity, $v_{j}$, at the the $j$ th surface node. As shown in Sec. III A, the structural impedance matrix $\mathbf{Z}_{s}$ is the dry impedance matrix and is independent of the mass loading from the fluid. To estimate $\mathbf{Z}_{s}$ by cross correlation, we multiply Eq. (2) by $\mathbf{p}^{H}$ to obtain the outer products

$$
\mathbf{Z}_{S}\left[\mathbf{v} \mathbf{p}^{H}\right]=\left[-\mathbf{p} \mathbf{p}^{H}\right]
$$

where the bracketed quantities are cross correlation, rank 1 matrices and superscript $H$ stands for the conjugate transpose. Because the impedance matrix is independent of the ensonification, we take the average of $L$ realizations $\mathbf{p}_{1}, \mathbf{p}_{2}, \ldots, \mathbf{p}_{L}$ to obtain expressions in terms of sample cross-correlation matrices

$$
\left\langle\mathbf{p} \mathbf{p}^{H}\right\rangle \equiv \frac{1}{L} \sum_{k=1}^{L} \mathbf{p}_{k} \mathbf{p}_{k}^{H}, \quad\left\langle\mathbf{v} \mathbf{p}^{H}\right\rangle \equiv \frac{1}{L} \sum_{k=1}^{L} \mathbf{v}_{k} \mathbf{p}_{k}^{H} .
$$

If we take the statistics of the ensonifying field to be Gaussian, then it has been shown that taking $L>3 N$ is sufficient to construct a full rank, well conditioned sample cross correlation matrix. ${ }^{15}$ Given full rank matrices, inversion is 
feasible and the impedance matrix can therefore be written as

$$
\mathbf{Z}_{s}=-\left\langle\mathbf{p p}^{H}\right\rangle\left\langle\mathbf{v} \mathbf{p}^{H}\right\rangle^{-1}
$$

Equation (6) suggests that we can measure the structural impedance matrix by placing the object in a random noise field and performing the prescribed cross correlations: On a set of sensors, cross correlate all measured pressures with each other and cross correlate all measured pressures with all measured normal velocities. This formulation is akin to the noise correlation methodology associated with extracting Green's functions. ${ }^{16-22}$ Typically, in the latter formulation, it is only the imaginary part of the Green's function that is extracted (i.e., the Green's function and its time-reversed form). However, in our formulation, we have the restriction that we are measuring the outward normal velocity, thereby specifying a direction. In fact, because the total fields and derivatives are at the surface nodes of the object, we must adequately excite the object at all these points to perform a stable inversion for the impedance matrix. Otherwise, we could use a limited noise distribution ${ }^{23,24}$ in which only a sector of sensors are used in a nondiffuse noise field.

Note that for a high compressibility (soft) object, the structural impedance matrix is a zero matrix $\mathbf{Z}_{s}=0,{ }^{25}$ whereas Eq. (6) is not defined if the surface velocity is zero due to the inversion in Eq. (6) as happens for a rigid object. In that latter case, the rigid object is characterized by a structural Green's function or a mobility matrix $\mathbf{Y}_{s}=0$, (inverse of the structural impedance matrix).

\section{STRUCTURAL IMPEDANCE FOR AN INFINITE CYLINDRICAL SHELL}

The correlation method for estimating the structural impedance matrix of a body in a random field is demonstrated numerically for an infinite cylindrical shell immersed in a fluid medium and filled with a fluid. First the analytical formulation of the structural impedance matrix is derived in Sec. III A. Then we show that the correlation process of Eq. (6) produces the same analytical expression. Finally, using a COSMOL Multiphysics numerical simulation, the structural impedance matrix is estimated following Eq. (6) and compared to the analytical results.

\section{A. Analytical expression of the structural impedance matrix}

We consider a body that has a circular cross section and use a modal approach to develop the structural impedance matrix. The external and inner radius of the shell are denoted $a$ and $b$, respectively. Using formulations from Doolittle and Überall, ${ }^{26}$ the scattered pressure field from an infinite cylindrical shell excited by a plane wave incident from an angle $\theta$ is

$$
p_{s}(r, \phi, \theta)=P_{i} \sum_{n=-\infty}^{+\infty}(-i)^{n} R_{n} H_{n}\left(k_{0} r\right) e^{i n(\phi-\theta)},
$$

which simplifies as

$$
p_{s}(r, \phi)=P_{i} \sum_{n=-\infty}^{+\infty} i^{n} R_{n} H_{n}\left(k_{0} r\right) e^{i n \phi} \quad \text { for } \theta=\pi
$$

where $P_{i}$ is the amplitude of the incident field, $k_{0}$ is the wavenumber in the fluid surrounding the shell, and $r$ and $\phi$ represent the polar coordinates of the receiver with the basis origin at the center of the shell. The function $H_{n}$ refers to the Hankel function of first kind $H_{n}^{(1)}$. The scattering coefficient of the shell $R_{n}$ is obtained from the boundary conditions between the shell and the inner and outer fluid and is defined as the ratio between two determinants ${ }^{26}$

$$
R_{n}=\frac{D_{n}^{[1]}}{D_{n}}
$$

The full expressions for the determinants $D_{n}$ and $D_{n}^{[1]}$ are given in Appendix A. Using Eq. (7b) and the modal decomposition of the incident plane wave at $\theta=\pi$,

$$
p_{i}(r, \phi)=P_{i} e^{i k r \cos \phi}=\sum_{n=-\infty}^{+\infty} i^{n} J_{n}\left(k_{0} a\right) e^{i n \phi}
$$

the total field at the boundary of the shell is

$$
p(a, \phi)=P_{i} \sum_{n=-\infty}^{\infty} i^{n}\left(J_{n}\left(k_{0} a\right)+R_{n} H_{n}\left(k_{0} a\right)\right) e^{i n \phi} .
$$

Although we have chosen a specific angle of incidence, $\theta=\pi$, the result in Eq. (9) is unchanged for an arbitrary angle of incidence due to the rotational symmetry of the shell. In the following, all results are for $\theta=\pi$.

The normal velocity at the shell boundary follows from Euler's equation,

$$
v(a, \phi)=\frac{P_{i}}{i \rho_{0} c_{0}} \sum_{n=-\infty}^{+\infty} i^{n}\left(J_{n}^{\prime}\left(k_{0} a\right)+R_{n} H_{n}^{\prime}\left(k_{0} a\right)\right) e^{i n \phi},
$$

where $c_{0}$ and $\rho_{0}$ are the sound speed and the density of the fluid surrounding the shell. Following Eqs. (9) and (10), we denote $p_{n}$ and $v_{n}$ the modal components of the decomposition of the total pressure field, $p(a, \phi)=\sum_{n=-\infty}^{\infty} p_{n}(a) e^{i n \phi}$, and normal velocity, $v(a, \phi)=\sum_{n=-\infty}^{\infty} v_{n}(a) e^{i n \phi}$. These components are computed using the forward Fourier transform

$$
p_{n}(a)=\frac{1}{2 \pi} \int p(a, \phi) e^{-i n \phi} d \phi \text {. }
$$

The ratio between the modal coefficients of the total pressure, Eq. (9), and the normal velocity, Eq. (10), yields the modal coefficients, $z_{s n}$, of the structural impedance matrix

$$
z_{s n}(a)=\frac{p_{n}(a)}{v_{n}(a)}=-i \rho_{0} c_{0} \frac{J_{n}\left(k_{0} a\right)+R_{n} H_{n}\left(k_{0} a\right)}{J_{n}^{\prime}\left(k_{0} a\right)+R_{n} H_{n}^{\prime}\left(k_{0} a\right)} .
$$

After a few manipulations (see Appendix A for more details), the modal coefficients of the structural impedance 
are expressed in terms of the shell and the inner medium properties

$$
z_{s n}(a)=i \frac{\rho_{1} c_{s}^{2}}{\omega a} \frac{D_{n}^{[21]}}{D_{n}^{[11]}}
$$

where full expressions of determinants $D_{n}^{[11]}$ and $D_{n}^{[21]}$ are given in Appendix B and, $c_{s}$ and $\rho_{1}$ are the shear speed and the density of the shell, respectively.

Although the total pressure field and normal velocity at the surface depend on the external medium properties, their ratio cancels out the impact of the external fluid loading and provides the structural or dry impedance. To illustrate this remark, the pressure and velocity from Eqs. (9) and (10) are reformulated in Eqs. (13) and (14) in terms of the structural impedance and the acoustic impedance. The acoustic impedance, discussed in Sec. IV, relates the scattered pressure to the scattered velocity and its modal coefficient for this cylindrical geometry is $z_{a n}(a)=i \rho_{0} c_{0}\left(H_{n}\left(k_{0} a\right) / H_{n}^{\prime}\left(k_{0} a\right)\right)$. Using this definition of the acoustic impedance and the modal coefficients of the structural impedance from Eq. (11) yields

$$
\begin{aligned}
& p(a, \phi)=\frac{2 P_{i}}{\pi k_{0} a} \sum_{n=-\infty}^{+\infty} \frac{i^{n+1}}{H_{n}^{\prime}\left(k_{0} a\right)} \frac{z_{s n}}{z_{s n}+z_{a n}} e^{i n \phi}, \\
& v(a, \phi)=-\frac{2 P_{i}}{\pi k_{0} a} \sum_{n=-\infty}^{+\infty} \frac{i^{n+1}}{H_{n}^{\prime}\left(k_{0} a\right)} \frac{1}{z_{s n}+z_{a n}} e^{i n \phi} .
\end{aligned}
$$

It becomes clear, given that the denominators of Eqs. (13) and (14) are identical, that the ratio of the modal components of the total pressure and the velocity removes the impact of the external fluid loading manifested through $z_{a n}$.

Finally, the structural impedance $\mathbf{Z}_{s}$ is calculated from the modal coefficient in Eq. (12) using the inverse Fourier transform

$$
Z_{S}(\phi)=\sum_{n=-\infty}^{\infty} z_{s n}(a) e^{i n \phi}
$$

and the convolution theorem with $\otimes$ representing the convolution

$$
p(a, \phi)=-\sum_{n=-\infty}^{\infty} z_{s n} v_{n} e^{i n \phi}=-\frac{1}{2 \pi} Z_{s}(\phi) \otimes v(a, \phi),
$$

which leads to

$$
p(a, \phi)=-\frac{1}{2 \pi} \int Z_{s}\left(\phi-\phi^{\prime}\right) v\left(a, \phi^{\prime}\right) d \phi^{\prime} .
$$

Discretization of Eq. (16) leads to the matrix equation, Eq. (3), $\mathbf{p}=-\mathbf{Z}_{s} \mathbf{v}$ where $\mathbf{p}$ and $\mathbf{v}$ are column vectors spanning the circumference of the shell. As before $\mathbf{Z}_{s}$ represents the properties of the shell and is independent of the parameters of the outer fluid or the forces acting on the shell. We also note that $\mathbf{Z}_{s}$ will be purely imaginary in the case of a lossless structure (see Appendix C for formal justification).

\section{B. Analytical derivation of the structural impedance matrix from the correlation process}

Analytically the structural impedance of Eq. (15) was determined from considering a plane wave incident from a single angle $\theta=\pi$. However, to measure $\mathbf{Z}_{s}$ from an experiment is a rather different matter as was pointed out in the discussion leading to Eq. (6). That is, the resulting matrix in the second equation of Eq. (5) is rank one and can not be inverted in Eq. (6) for a single angle of incidence. Thus we turn to multiple angles of incidence and use the formulation of solving for correlations ${ }^{27,28}$ to obtain the structural impedance using the correlation process presented in Sec. II. Starting with Eq. (9) for an incident plane wave with an arbitrary angle of incidence $\theta_{1}$, the pressure field at angle $\phi_{1}$ is

$p\left(a, \phi_{1}, \theta_{1}\right)=P_{1 i} \sum_{n=-\infty}^{+\infty}(-i)^{n}\left(J_{n}\left(k_{0} a\right)+R_{n} H_{n}\left(k_{0} a\right)\right) e^{i n\left(\phi_{1}-\theta_{1}\right)}$.

Then we consider the cross correlation of the pressure,

$$
\begin{aligned}
p\left(a, \phi_{1}, \theta_{1}\right) p^{*}\left(a, \phi_{2}, \theta_{2}\right) & \\
= & P_{1 i} P_{2 i}^{*} \sum_{m=-\infty}^{+\infty} \sum_{n=-\infty}^{+\infty}\left(-i^{m}\right)\left(i^{n}\right) e^{i m\left(\phi_{1}-\theta_{1}\right)} e^{-i n\left(\phi_{2}-\theta_{2}\right)} \\
& \times\left[J_{m}\left(k_{0} a\right)+R_{m} H_{m}\left(k_{0} a\right)\right]\left[J_{n}^{*}\left(k_{0} a\right)+R_{n}^{*} H_{n}^{*}\left(k_{0} a\right)\right]
\end{aligned}
$$

where $\theta_{2}$ and $\phi_{2}$ refer to an other arbitrary incident angle and other field angle. We now treat the incident fields as random uncorrelated plane waves and integrate over all possible directions to obtain the cross correlation of the pressure on the surface of the object in a homogeneous plane wave noise field,

$$
\begin{aligned}
& \left\langle p\left(a, \phi_{1}, \theta_{1}\right) p^{*}\left(a, \phi_{2}, \theta_{2}\right)\right\rangle \\
& =\int d \theta_{1} \int d \theta_{2}\left\langle P_{1 i} P_{2 i}^{*}\right\rangle \\
& \quad \times \sum_{m=-\infty}^{+\infty} \sum_{n=-\infty}^{+\infty}\left(-i^{m}\right)\left(i^{n}\right) e^{i m\left(\phi_{1}-\theta_{1}\right)} e^{-i n\left(\phi_{2}-\theta_{2}\right)} \\
& \quad \times\left[J_{m}\left(k_{0} a\right)+R_{m} H_{m}\left(k_{0} a\right)\right]\left[J_{n}^{*}\left(k_{0} a\right)+R_{n}^{*} H_{n}^{*}\left(k_{0} a\right)\right]
\end{aligned}
$$

For a homogeneous plane wave random noise field, we have

$$
\left\langle P_{1 i} P_{2 i}^{*}\right\rangle=|P|^{2} \delta\left(\theta_{1}-\theta_{2}\right)
$$

Integrating over $\theta_{2}$ then $\theta_{1}$ (integration of exponent in $\theta_{1}$ yields $\left.2 \pi \delta_{m n}\right)$, we get

$$
\begin{aligned}
& \left\langle p\left(a, \phi_{1}\right) p^{*}\left(a, \phi_{2}\right)\right\rangle \\
& =2 \pi|P|^{2} \sum_{n=-\infty}^{+\infty} e^{i n\left(\phi_{1}-\phi_{2}\right)} \\
& \quad \times\left[J_{n}\left(k_{0} a\right)+R_{n} H_{n}\left(k_{0} a\right)\right]\left[J_{n}^{*}\left(k_{0} a\right)+R_{n}^{*} H_{n}^{*}\left(k_{0} a\right)\right] .
\end{aligned}
$$


Furthermore following the same exact procedure with Euler's equation, we get from Eq. (10),

$$
\begin{aligned}
\left\langle v\left(a, \phi_{1}\right) p^{*}\left(a, \phi_{2}\right)\right\rangle & \\
= & 2 \pi \frac{|P|^{2}}{i \rho_{0} c_{0}} \sum_{n=-\infty}^{+\infty} e^{i n\left(\phi_{1}-\phi_{2}\right)} \\
& \times\left[J_{n}^{\prime}\left(k_{0} a\right)+R_{n} H_{n}^{\prime}\left(k_{0} a\right)\right]\left[J_{n}^{*}\left(k_{0} a\right)+R_{n}^{*} H_{n}^{*}\left(k_{0} a\right)\right] .
\end{aligned}
$$

The ratio between the modal coefficients of Eqs. (21) and (22) yields the same result as Eq. (11),

$$
z_{s n}=-i \rho_{0} c_{0} \frac{J_{n}\left(k_{0} a\right)+R_{n} H_{n}\left(k_{0} a\right)}{J_{n}^{\prime}\left(k_{0} a\right)+R_{n} H_{n}^{\prime}\left(k_{0} a\right)} .
$$

Again the structural impedance $\mathbf{Z}_{s}$ is calculated from the modal coefficient in Eq. (23) using the inverse transform, Eq. (15), and the convolution theorem

$$
\begin{aligned}
& \left\langle p\left(a, \phi_{1}\right) p^{*}\left(a, \phi_{2}\right)\right\rangle \\
& \quad=-\frac{1}{2 \pi} \int Z_{s}\left(\phi_{2}-\phi^{\prime}\right)\left\langle v\left(a, \phi_{1}\right) p^{*}\left(a, \phi^{\prime}\right)\right\rangle d \phi^{\prime} .
\end{aligned}
$$

Discretizing formulation (24) and defining vectors $\mathbf{p}$ and $\mathbf{v}$ spanning the whole circumference of the shell yields

$$
\left\langle\mathbf{p} \mathbf{p}^{H}\right\rangle=-\mathbf{Z}_{S}\left\langle\mathbf{v} \mathbf{p}^{H}\right\rangle
$$

We have therefore shown that the modal formulation of an infinite cylindrical shell in a homogeneous random noise field yields the structural impedance matrix as the discretized formulation Eq. (6). We proceed to demonstrate this method with a numerical experiment.

\section{Numerical experiment for the evaluation of the structural impedance matrix}

The method for estimating the structural impedance matrix for an elastic body in a random field is numerically demonstrated for an aluminum cylindrical shell filled with air and immersed in water. In this example, the aluminum shell has a Young's modulus $E_{1}=70 \mathrm{GPa}$, a Poisson ratio $\mu_{1}=0.33$, and density $\rho_{1}=2700 \mathrm{~kg} / \mathrm{m}^{3}$. The sound speeds in the external and inner medium are $c_{0}=1500 \mathrm{~m} / \mathrm{s}$ and $c_{2}=343 \mathrm{~m} / \mathrm{s}$, respectively; and the densities are $\rho_{0}=1000 \mathrm{~kg} / \mathrm{m}^{3}$ and $\rho_{2}=1.25 \mathrm{~kg} / \mathrm{m}^{3}$. The inner and outer radius of the shell are $b=21.5 \mathrm{~cm}$ and $a=22.5 \mathrm{~cm}$, respectively. The structural impedance matrix is evaluated at $2 \mathrm{kHz}$.

The random field consists of the incoherent superposition of plane waves of all directions and phases. ${ }^{16}$ Thus the pressure field and normal velocity at the $N$ nodes of the shell surface are computed over $M$ realizations, each realization associated to a distinct incident plane wave with a Gaussian phase and amplitude distribution. Taking advantage of the cylindrical shell symmetry, we construct by rotation $N$ realizations of the pressure field and velocity field at the $N$ surface nodes of the shell surface from a single simulation. To get the pressure field and normal velocity at $\mathrm{N}$ surface nodes, we perform $L$ simulations with the finite element software COMSOL Multiphysics as a tool to simulate an experiment, leaving $M=L \times N$ realizations. The finite element simulation was performed with $N=400$ surface nodes evenly spaced and for $L=400$ incident plane waves. Then we compute the structural impedance matrix from 100 surface nodes (evenly spaced and chosen among the 400 existing surface nodes). The sample cross-correlation matrices $\left\langle\mathbf{p} \mathbf{p}^{H}\right\rangle$ and $\left\langle\mathbf{v} \mathbf{p}^{H}\right\rangle$ are computed by averaging over 5000 realizations (chosen randomly over the $M=160000$ realizations). Then, the inverse of the factor $\left\langle\mathbf{v p}{ }^{H}\right\rangle$ is computed using a singular value decomposition (SVD). Figure 2 shows the singular values of the factor $\left\langle\mathbf{v p}{ }^{H}\right\rangle$, where 18 singular values are significant with the 20th singular value falling into the computational noise, indicated by the broad plateau starting at $-120 \mathrm{~dB}$. Note the short steps in the curve before this plateau indicate a degeneracy in the modes that is entirely expected. That is for any mode $n$, there exists $\cos (n \phi)$ and $\sin (n \phi)$ components that are degenerate for our axisymmetric shell and that the SVD interprets as equal level singular values creating the short steps in the curve in Fig. 2. Due to this degeneracy, the number of singular values is double the number of modes.

First, $\mathbf{Z}_{s}$ is computed [Fig. 3(a)] for a lossless shell using SVD with 18 eigenvalues. Note that the magnitude of the real part is 500 times below the magnitude of the imaginary part of $\mathbf{Z}_{s}$; thus this non zero real part has no impact on the scattered field calculation and is negligible. This result is in a good agreement with the analytical calculation of $\mathbf{Z}_{S}$ [Fig. 3(b)], which is purely imaginary for the lossless case as demonstrated in Appendix C.

Finally, Fig. 4 compares the analytical and numerical structural impedance matrix when damping is introduced to the body, $\eta_{1}=0.05$. Both results are in a good agreement, and we note that the impedance is now a complex number with a non zero real part. Indeed, the damping factor, ${ }^{14} \eta_{1}$, introduces a complex Young modulus (or complex compressional and shear wave speeds) so that the structural impedance of the object is not purely imaginary.

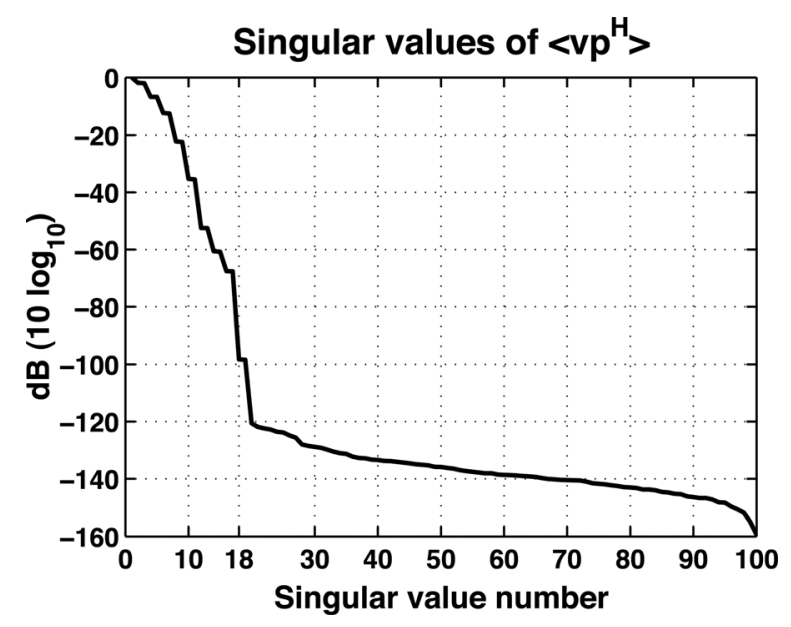

FIG. 2. Singular values of the operator $\left\langle\mathbf{v} \mathbf{p}^{H}\right\rangle$. 
(a)
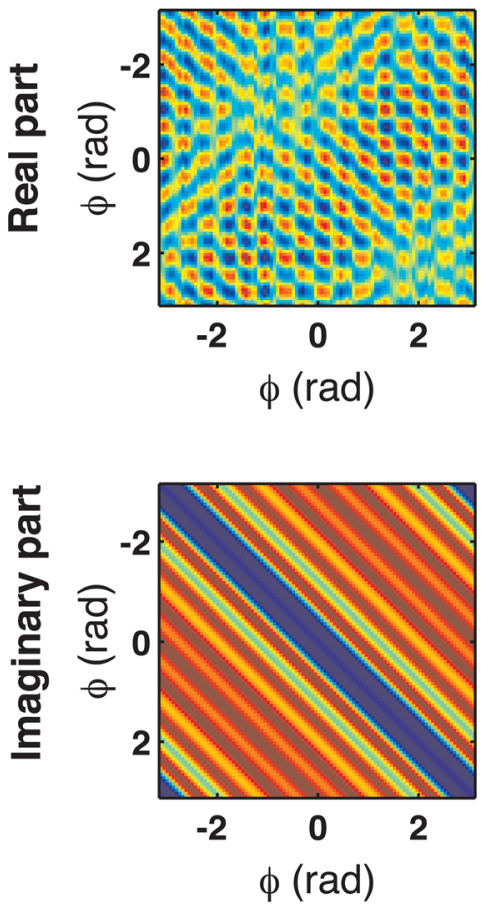

(b)
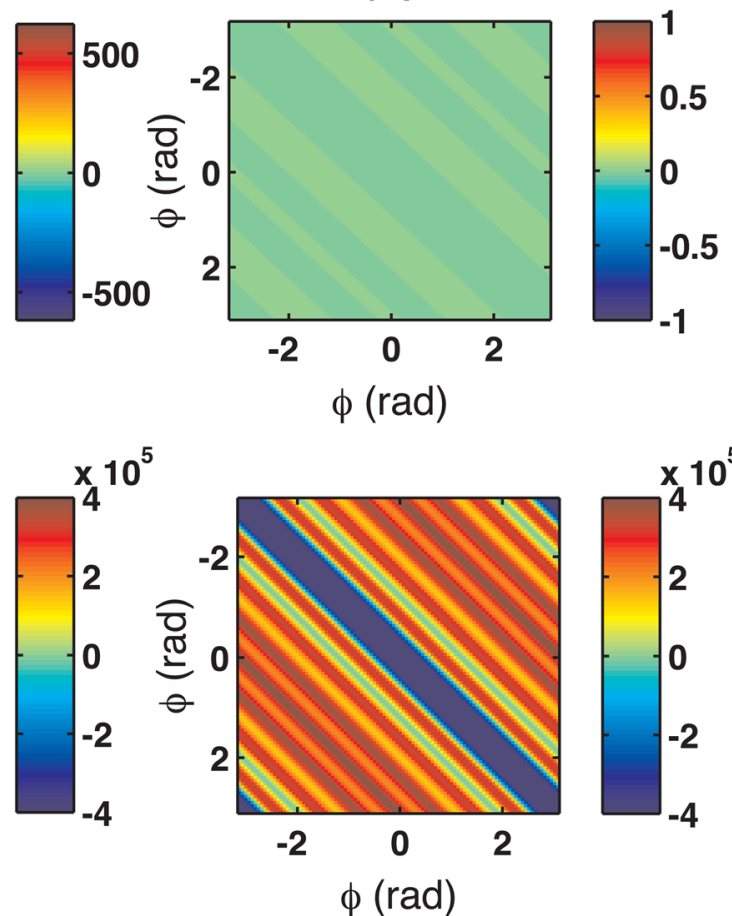

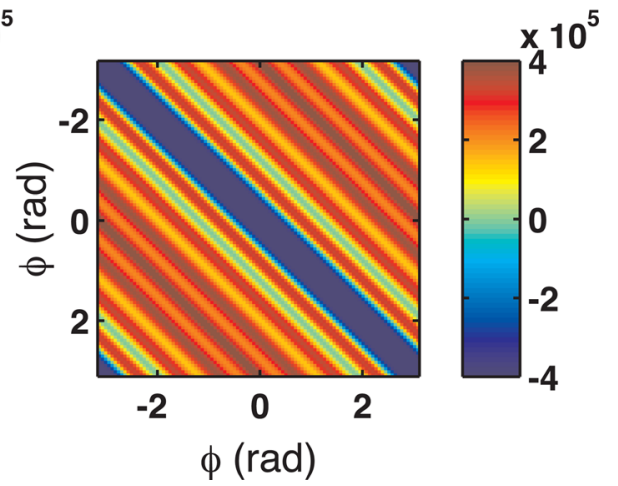

FIG. 3. (Color online) Structural impedance matrix ( $\mathrm{Pa} \mathrm{s} / \mathrm{m})$ at 100 surface nodes for a lossless cylindrical shell. (a) Numerical result with pseudoinversion of $\left\langle\mathbf{v} \mathbf{p}^{H}\right\rangle$ performed with the 18 largest eigenvalues; note that the magnitude of the real part is 500 times below the magnitude of the imaginary part of $\mathbf{Z}_{s}$; thus this non zero real part has no impact on the scattered field calculation. (b) Analytical solution using nine modes.
The next and crucial step in our analytic example of the correlation method is to use the computed $\mathbf{Z}_{s}$ to predict the scattered field from the cylinder given any incident field of our choosing. As discussed in the next section, this requires a knowledge of two other impedance matrices: the internal impedance matrix and the acoustic impedance matrix. We postpone our result for the scattered field until Sec. IV B to discuss the general impedance formulations.

\section{FORMULATIONS FOR THE PREDICTION OF THE SCATTERED FIELD USING THE MEASUREMENT OF THE STRUCTURAL IMPEDANCE MATRIX}

We want to show that when the impedance matrix $\mathbf{Z}_{S}$ is known, one can predict the scattered field from an object from just the specification of the incident pressure field over the surface. This prediction is carried out using the equivalent source method (ESM). ${ }^{5,29-31}$ In the literature, the ESM
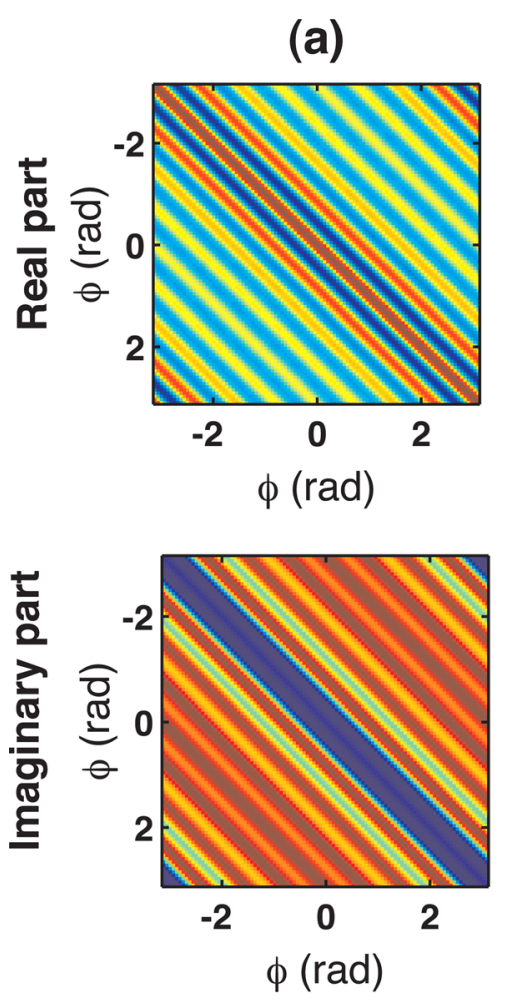

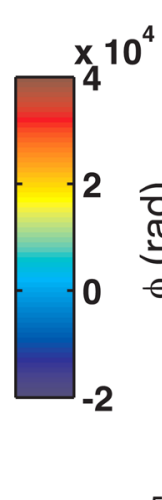

(b)

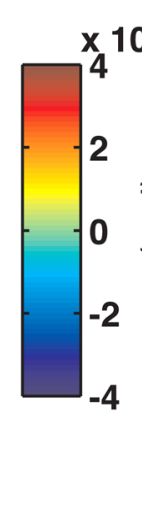

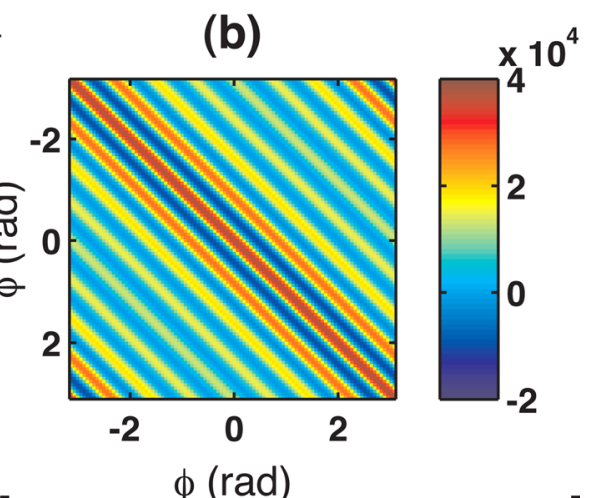

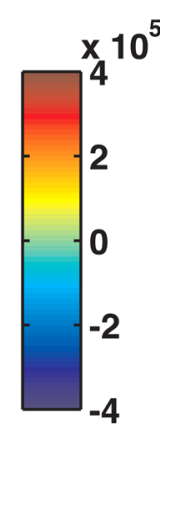

FIG. 4. (Color online) Structural impedance matrix $(\mathrm{Pa} \mathrm{s} / \mathrm{m})$ at 100 surface nodes for a lossy cylindrical shell with damping $\eta_{1}=0.05$. (a) Numerical result with pseudo-inversion of $\left\langle\mathbf{v p}^{H}\right\rangle$ performed with the 18 largest eigenvalues. (b) Analytical solution using nine modes. 
is also referred to as the wave superposition method or the virtual source method.

Central to this approach are three linear matrix equations

$$
\begin{aligned}
& \mathbf{p}_{i}=-\mathbf{Z}_{i} \mathbf{v}_{i} \\
& \mathbf{p}_{s}=-\mathbf{Z}_{a} \mathbf{v}_{s} \\
& \mathbf{p}_{i}+\mathbf{p}_{s}=-\mathbf{Z}_{s}\left(\mathbf{v}_{i}+\mathbf{v}_{s}\right)
\end{aligned}
$$

Equation (26) describes the relationship between the incident pressure and incident normal velocity fields on the surface $\Omega$, where $\mathbf{Z}_{i}$ is the internal field impedance or the incident impedance. ${ }^{1}$ The acoustic impedance matrix $\mathbf{Z}_{a}$ in Eq. (27) relates the scattered pressure field to scattered velocity field on the surface. In radiation problems with no exterior fields, $\mathbf{Z}_{a}$ relates surface vibration induced by internal forces to the resulting surface pressure and in this case is called the radiation impedance. ${ }^{1}$ In either case, $\mathbf{Z}_{a}$ represents the loading of the outer fluid on the surface. Finally, the last of the three matrix equations, Eq. (28), is a restatement of Eq. (3). The three impedance matrices, $\mathbf{Z}_{i}, \mathbf{Z}_{a}, \mathbf{Z}_{s}$, are denoted $\mathbf{Z}_{i}, \mathbf{Z}_{r}, \mathbf{Z}$, respectively, in the paper by Bobrovnitskii. ${ }^{1}$

Simple manipulation of Eqs. (27) and (28) to eliminate $\mathbf{v}_{s}$ leads to a fundamental relationship between the scattered pressure on the surface and the incident field there,

$$
\mathbf{p}_{s}=-\mathbf{Z}_{a}\left(\mathbf{Z}_{a}+\mathbf{Z}_{s}\right)^{-1}\left(\mathbf{p}_{i}+\mathbf{Z}_{s} \mathbf{v}_{i}\right) .
$$

Given that the impedance matrices are invertible, we can remove $\mathbf{v}_{i}$ from Eq. (29) using Eq. (26), to obtain

$$
\mathbf{p}_{s}=-\mathbf{Z}_{a}\left(\mathbf{Z}_{a}+\mathbf{Z}_{s}\right)^{-1}\left(\mathbf{I}+\mathbf{Z}_{s} \mathbf{Z}_{i}^{-1}\right) \mathbf{p}_{i} \text {. }
$$

Unlike $\mathbf{Z}_{s}$, we do not measure $\mathbf{Z}_{a}$ and $\mathbf{Z}_{i}$; rather, we compute them numerically using the ESM method as described next.

Alternatively, when defining the inverse of each impedance matrix as $\mathbf{Y}_{\alpha}=\mathbf{Z}_{\alpha}^{-1}$ where $\alpha$ is any of the three subscripts, we obtain the simple form relating the scattered pressure to the incident pressure presented by Bobrovnitskii, ${ }^{1}$

$$
\mathbf{p}_{s}=\left(\mathbf{Y}_{a}+\mathbf{Y}_{s}\right)^{-1}\left(\mathbf{Y}_{i}-\mathbf{Y}_{s}\right) \mathbf{p}_{i}
$$

\section{A. Derivation of the scattered field using ESM}

The acoustic impedance matrix $\mathbf{Z}_{a}$, as well as $\mathbf{Z}_{i}$, depend only upon the geometry of $\Omega$ and the Greens's function in the external fluid that satisfies the boundary conditions at remote boundaries, such as an ocean surface, walls in a room, etc. For an infinite space above the scatterer, we use the well known free-space Green's function,

$$
G(\mathbf{y}, \mathbf{x}) \equiv e^{i k|\mathbf{y}-\mathbf{x}|} / 4 \pi|\mathbf{y}-\mathbf{x}|,
$$

where $\mathbf{x}$ is the vector to the source point and $\mathbf{y}$ the vector to the field point.

We derive $\mathbf{Z}_{a}$ using the equivalent source method which replaces the body with a conformal set of point sources with unknown strengths represented in a column vector $\mathbf{s}$ that are located just inside $\Omega$ (Fig. 5). We place a set of field points $y_{i}$

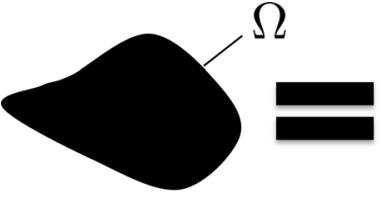

Scattering object

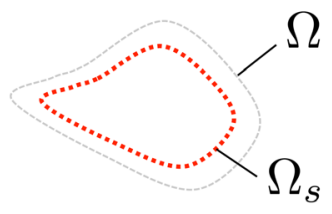

Set of $N$ equivalent point sources
FIG. 5. (Color online) The equivalent source method replaces the scattering object by a set of virtual point sources located at $\Omega_{s}$ just inside the surface of the object $\Omega$.

uniformly distributed on $\Omega$ and place the conformal set of source points at locations $x_{j}$ to yield the matrix relating the $i$ th surface node to the $j$ th point source,

$$
p_{s}\left(y_{i}\right)=i \omega \rho_{0} \sum_{j} G\left(y_{i}, x_{j}\right) s\left(x_{j}\right),
$$

and define the elements of a matrix $\mathbf{M}_{c}$ to be $i \omega \rho_{0} G\left(y_{i}, x_{j}\right)$ leading to

$$
\mathbf{p}_{s}=\mathbf{M}_{c} \mathbf{s}
$$

From Eq. (33), the normal velocity on the surface is obtained using Euler's equation

$$
\mathbf{v}_{s}=\mathbf{D}_{c} \mathbf{s}
$$

where the elements of $\mathbf{D}_{c}$ are $\partial_{n\left(y_{i}\right)} G\left(y_{i}, x_{j}\right)$, the normal derivative of the Green's function taken with respect to the field point. Solving Eq. (35) for $\mathbf{s}$ and inserting into Eq. (34) immediately provides the acoustic impedance matrix $\mathbf{Z}_{a}$ of Eq. (27),

$$
\mathbf{Z}_{a}=\mathbf{M}_{c} \mathbf{D}_{c}^{-1}
$$

Using Eqs. (34) and (29), the unknown source strength vector is

$$
\mathbf{s}=-\mathbf{D}_{c}^{-1}\left(\mathbf{Z}_{a}+\mathbf{Z}_{s}\right)^{-1}\left(\mathbf{p}_{i}+\mathbf{Z}_{s} \mathbf{Z}_{i}^{-1} \mathbf{p}_{i}\right)
$$

This formulation is equivalent to the virtual source strength formulation given in the paper by Lucifredi [Eq. (4)]. ${ }^{4}$

Once $\mathbf{s}$ is known, the scattered field $\mathbf{p}_{\text {so }}$ can be computed at any point or set of points $\mathbf{z}$ outside $\Omega$ by replacing the field point $\mathbf{y}$ with $\mathbf{z}$ in Eq. (33) and using Eq. (37),

$$
\mathbf{p}_{s o}=-\mathbf{M}_{\mathrm{zi}} \mathbf{D}_{c}^{-1}\left(\mathbf{Z}_{a}+\mathbf{Z}_{s}\right)^{-1}\left(\mathbf{I}+\mathbf{Z}_{s} \mathbf{Z}_{i}^{-1}\right) \mathbf{p}_{i},
$$

where the elements of $\mathbf{M}_{z i}$ are $i \omega \rho_{0} G\left(z_{i}, x_{j}\right)$.

Calculation of $\mathbf{Z}_{i}$ follows in a similar manner to Eq. (33), however, a different set of sources $\mathbf{s}_{i}$ are placed just outside the surface with the interior volume filled with the exterior fluid (the exterior fluid is replaced with a vacuum as shown in Fig. 1) and the following calculation is made:

$$
p_{i}\left(y_{i}\right)=i \omega \rho_{0} \sum_{j} G\left(y_{i}, x_{j}\right) s_{i}\left(x_{j}\right),
$$

leading, in similar fashion to Eq. (34)-(36), to $\mathbf{Z}_{i}$. 
Equation (38) shows the power of the approach. All the quantities are known except the incident field $\mathbf{p}_{i}$, which we are free to choose to represent any desired source condition needed to be investigated. Whereas $\mathbf{M}_{c}$ and $\mathbf{D}_{c}$ are not functions of the interior elastic parameters, $\mathbf{Z}_{s}$ provides the interface conditions and introduces the effect of scattering from the elastic effects of the interior body. Exterior boundaries can be introduced by using a Green's function in Eq. (32) that accounts for them and the formulation remains the same except for the change in $\mathbf{G}$ in Eq. (32). For example, a submerged body below an air-water interface would require a Green's function that included images across this boundary to satisfy the Dirichlet boundary condition at the interface. In the work by Lucifredi, ${ }^{4}$ Green's functions were used for a stratified medium arriving at an expression identical to Eq. (37).

\section{B. Prediction of the scattered field from the noise correlation numerical experiment}

As an illustration, the ESM is applied to compute the scattered field by the cylindrical shell from the evaluation of its structural impedance matrix. We consider the lossy shell example presented in Sec. IIIC. The lossy shell is immersed in water in an infinite medium. The scattered field is computed from the numerical estimation of the structural impedance matrix over 100 nodes (see Sec. III C and Fig. 4). Thus the shell structure is represented by 100 equivalent sources. The equivalent sources are located inside the boundary of the shell but in the outer fluid medium at a distance $0.6 d_{n}$ from the shell surface, ${ }^{4} d_{n}$ being the lattice spacing. To calculate the equivalent source strengths, Eq. (37), one needs to compute the acoustic impedance $\mathbf{Z}_{a}$ and the incident impedance $\mathbf{Z}_{i}$ to get the incident velocity from the incident pressure field given the relation $\mathbf{p}_{i}=\mathbf{Z}_{i} \mathbf{v}_{i}$. We compute $\mathbf{Z}_{a}$ and $\mathbf{Z}_{i}$ from relation (36) using the two-dimensional (2D) free field Green's function in water,

$$
G(\mathbf{y}, \mathbf{x})=\frac{i}{4} H_{0}^{(1)}\left(k_{0}|\mathbf{y}-\mathbf{x}|\right)
$$

to define the elements of the Green's function matrix $\mathbf{M}_{c}$ and the normal derivative matrix $\mathbf{D}_{c}$, where rows and columns correspond to the source points $\mathbf{x}$ and the surface nodes $\mathbf{y}$, respectively. Determination of $\mathbf{Z}_{a}$ and $\mathbf{Z}_{i}$ is very similar, the difference lies in the position of the source points $\mathbf{y}$ that are just inside the shell (equivalent source locations at a distance $0.6 d_{n}$ from the shell surface) for $\mathbf{Z}_{a}$ and outside the shell for calculation of $\mathbf{Z}_{i}$. Once the source strengths are known, the scattered field is computed at any set of points outside the shell using the free-field Green's function, Eq. (40), between the equivalent sources and the observation points. Figure 6 compares the scattered pressure field at a radius of $40 \mathrm{~cm}$ from the equivalent source method with the exact solution when the cylindrical shell is excited by a $2 \mathrm{D}$ point source at $2 \mathrm{kHz}$ located $3 \mathrm{~m}$ from the shell center. The ESM and analytical solutions are in a very good agreement for this near-field configuration.
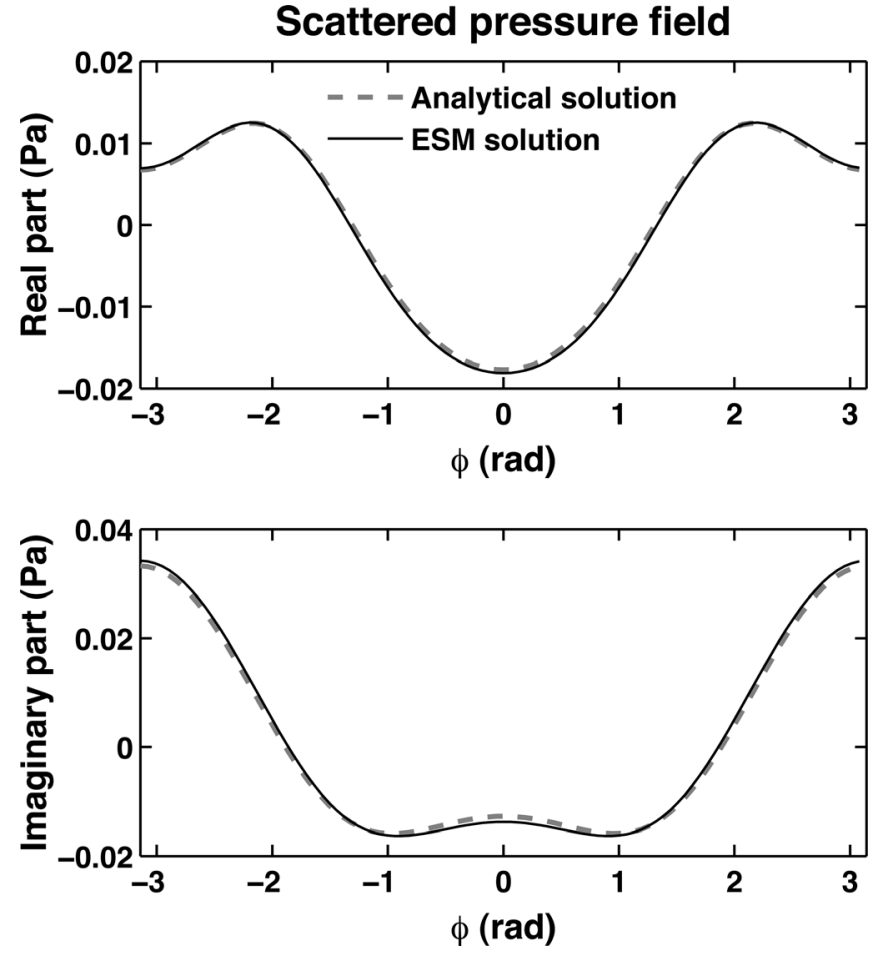

FIG. 6. Comparison of the scattered pressure fields from the equivalent source method (ESM) with the analytical solution when the cylindrical shell is excited by a $2 \mathrm{D}$ point source at $2 \mathrm{kHz}$. The point source is located at $3 \mathrm{~m}$ from the center of the shell. The scattered pressure field is plotted for receivers located all around the shell at $0.4 \mathrm{~m}$ from the center of the shell. The ESM solution uses 100 equivalent sources and the structural impedance matrix (Fig. 4) numerically evaluated using the correlation process.

\section{DISCUSSION}

The efficacy of the structural impedance evaluation relies on the number of realizations for computing expectation value of the correlations $\left\langle\mathbf{p} \mathbf{p}^{H}\right\rangle$ and $\left\langle\mathbf{v} \mathbf{p}^{H}\right\rangle$, the number of surface nodes, and the number of singular values used for inverting $\left\langle\mathbf{v} \mathbf{p}^{H}\right\rangle$. To get an accurate estimate of the structural impedance matrix, we assume a large number of realizations to properly construct the cross-correlation matrices (at least $L>3 N$ as mentioned in Sec. II).

Varying the number of surface nodes and the number of eigenvalues affects the resolution of the structural impedance matrix, which will affect the accuracy of the scattered field computation using the ESM. The number of surface nodes and singular values to consider is dictated by a better than Nyquist sampling of the highest order surface mode excited at the highest frequency of interest. ${ }^{32}$ The distribution of point sources for the ESM method must also satisfy the same Nyquist condition. In the cylindrical shell example, the maximum number of significant singular values is 18 (Fig. 2). This means that at least 18 surface nodes are required to compute $\mathbf{Z}_{s}$. Moreover, the number of singular values to consider for the inversion of $\left\langle\mathbf{v} \mathbf{p}^{H}\right\rangle$ depends on the signal to noise ratio of the pressure and normal velocity data fields. The estimation of $\mathbf{Z}_{s}$ is more accurate when increasing the number of eigenvalues for the inversion using a SVD as long as the singular values are above the uncorrelated noise level as is the case for any matrix pseudo-inversion. 
Regarding the prediction of the scattered field using the ESM, this calculation is also affected by potential mismatch between the Green's function of the outer medium and the real environment. The effect of mismatch between the Green's function model and the outer environment is not discussed here as it is out of the scope of the paper. However, the reader can refer to the literature ${ }^{4,5,7}$ for more discussions on that topic.

\section{CONCLUSIONS}

This paper presented a method for obtaining the structural impedance matrix from a random field. The approach relies on the correlation between pressure and normal velocity noise field data at a given set of surface nodes at the body surface. Numerical results with an infinite cylindrical shell show very good agreement with the analytical solution. This structural impedance matrix is used for computing the scattered field either from the Helmholtz integral equation or the equivalent source method formulations. For both methods, computation of the scattered field involves two other impedances matrices, the acoustic impedance matrix and the incident impedance matrix. Only the ESM approach was discussed here because the HIE method is equivalent. This work finds applications for computing the scattered field of a complex object in any complex environment from the knowledge of its structural impedance matrix, the latter measured using a random noise field.

\section{ACKNOWLEDGMENT}

We acknowledge support from the Office of Naval Research.

\section{APPENDIX A: DEFINITION OF DETERMINANT $D_{n}$ AND $D_{n}^{[1]}$}

We consider scattering from an infinite cylindrical shell excited by a plane wave. The scattering coefficient $R_{n}$ for an infinite cylindrical shell is defined as the ratio between two determinants $^{26}$

$$
R_{n}=\frac{D_{n}^{[1]}}{D_{n}}
$$

where expression of determinant $D_{n}$ is ${ }^{33}$

$$
\begin{aligned}
D_{n} & =\operatorname{det}\left(\mathbf{D}_{\mathbf{n}}\right) \\
& =\left|\begin{array}{ccccc}
\alpha_{11}(a) & \alpha_{12}(a) & \cdots & \alpha_{15}(a) & 0 \\
\alpha_{21}(a) & \alpha_{22}(a) & \cdots & \alpha_{25}(a) & 0 \\
0 & \alpha_{32}(a) & \cdots & \alpha_{35}(a) & 0 \\
0 & \alpha_{12}(b) & \cdots & \alpha_{15}(b) & \alpha_{46}(b) \\
0 & \alpha_{22}(b) & \cdots & \alpha_{25}(b) & \alpha_{56}(b) \\
0 & \alpha_{32}(b) & \cdots & \alpha_{35}(b) & 0
\end{array}\right| .
\end{aligned}
$$

In the following, coefficients of the determinant $D_{n}$ are expressed in terms of the Bessel $J_{n}$, Hankel $H_{n}$, and Neuman $Y_{n}$ functions and variables $x_{p}$ and $x_{s}$. They are defined as $x_{p}=\omega r / c_{p}$ and $x_{s}=\omega r / c_{s}$, respectively, where $c_{p}$ is the compressional wave speed and $c_{s}$ the shear wave speed in the shell. The spatial variable $r$ is either equal to the outer radius $a$ or the inner radius $b$ of the shell.

$$
\begin{aligned}
& \alpha_{12}(r)=\left(x_{s}^{2}-2 n^{2}\right) J_{n}\left(x_{p}\right)+2 x_{p} J_{n}^{\prime}\left(x_{p}\right), \\
& \alpha_{13}(r)=\left(x_{s}^{2}-2 n^{2}\right) Y_{n}\left(x_{p}\right)+2 x_{p} Y_{n}^{\prime}\left(x_{p}\right), \\
& \alpha_{14}(r)=-2 n\left[J_{n}\left(x_{s}\right)-x_{s} J_{n}^{\prime}\left(x_{s}\right)\right], \\
& \alpha_{15}(r)=-2 n\left[Y_{n}\left(x_{s}\right)-x_{s} Y_{n}^{\prime}\left(x_{s}\right)\right], \\
& \alpha_{22}(r)=-x_{p} J_{n}^{\prime}\left(x_{p}\right), \\
& \alpha_{23}(r)=-x_{p} Y_{n}^{\prime}\left(x_{p}\right), \\
& \alpha_{24}(r)=n J_{n}\left(x_{s}\right), \\
& \alpha_{25}(r)=n Y_{n}\left(x_{s}\right), \\
& \alpha_{32}(r)=-2 n\left[J_{n}\left(x_{p}\right)-x_{p} J_{n}^{\prime}\left(x_{p}\right)\right], \\
& \alpha_{33}(r)=-2 n\left[Y_{n}\left(x_{p}\right)-x_{p} Y_{n}^{\prime}\left(x_{p}\right)\right], \\
& \alpha_{34}(r)=\left(x_{s}^{2}-2 n^{2}\right) J_{n}\left(x_{s}\right)+2 x_{s} J_{n}^{\prime}\left(x_{s}\right), \\
& \alpha_{35}(r)=\left(x_{s}^{2}-2 n^{2}\right) Y_{n}\left(x_{s}\right)+2 x_{s} Y_{n}^{\prime}\left(x_{s}\right) .
\end{aligned}
$$

The elements of the last column in Eq. (A2) depend on the inner medium sound speed $c_{2}$ and density $\rho_{2}$,

$$
\begin{aligned}
& \alpha_{46}(r)=x_{s}^{2} J_{n}\left(x_{2}\right), \\
& \alpha_{56}(r)=-\frac{\rho_{1}}{\rho_{2}} x_{2} J_{n}^{\prime}\left(x_{2}\right),
\end{aligned}
$$

where $x_{2}$ is defined as $x_{2}=\omega r / c_{2}$.

The elements of the first column in Eq. (A2) depend on the external medium sound speed $c_{0}$ and density $\rho_{0}$,

$$
\begin{aligned}
& \alpha_{11}(r)=x_{s}^{2} H_{n}\left(x_{0}\right), \\
& \alpha_{21}(r)=-\frac{\rho_{1}}{\rho_{0}} x_{0} H_{n}^{\prime}\left(x_{0}\right),
\end{aligned}
$$

where $x_{0}$ is defined as $x_{0}=\omega r / c_{0}$.

Definition of the determinant $D_{n}^{[1]}$ is the same as $D_{n}$ except that the elements of the first column $\alpha_{11}(a)$ and $\alpha_{21}(a)$ are replaced by $\beta_{1}(a)$ and $\beta_{2}(a)$,

$$
\begin{aligned}
& \beta_{1}(r)=-x_{s}^{2} J_{n}\left(x_{0}\right), \\
& \beta_{2}(r)=-\frac{\rho_{1}}{\rho_{0}} x_{0} J_{n}^{\prime}\left(x_{0}\right) .
\end{aligned}
$$

\section{APPENDIX B: DERIVATION OF $\boldsymbol{z}_{s n}$}

This section derives a formulation of the structural impedance, which only depends on the cylindrical shell and inner medium properties. As shown in Sec. III A, the modal coefficient of the structural impedance matrix is

$$
z_{s n}(a, \phi)=-i \rho_{0} c_{0} \frac{J_{n}\left(k_{0} a\right)+R_{n} H_{n}\left(k_{0} a\right)}{J_{n}^{\prime}\left(k_{0} a\right)+R_{n} H_{n}^{\prime}\left(k_{0} a\right)} .
$$

Expressing the determinant in terms of the first column elements $\alpha_{11}(a), \alpha_{21}(a), \beta_{1}(a)$, and $\beta_{2}(a)$ yields the following expression of the scattering coefficient 


$$
R_{n}=-\frac{\rho_{0} c_{0} x_{s}^{2} J_{n}\left(k_{0} a\right) D_{n}^{[11]}-\rho_{1} \omega a J_{n}^{\prime}\left(k_{0} a\right) D_{n}^{[21]}}{\rho_{0} c_{0} x_{s}^{2} H_{n}\left(k_{0} a\right) D_{n}^{[11]}-\rho_{1} \omega a H_{n}^{\prime}\left(k_{0} a\right) D_{n}^{[21]}}
$$

where $D_{n}^{[11]}$ and $D_{n}^{[21]}$ are related to the minor of matrix $\mathbf{D}_{\mathbf{n}}$ by eliminating row 1 and column 1, and row 2 and column 1 , respectively. The determinants $D_{n}^{[11]}$ and $D_{n}^{[21]}$ only depend on the shell and inner medium properties. Their full expressions are

$$
D_{n}^{[11]}=\left|\begin{array}{cccc}
\alpha_{22}(a) & \cdots & \alpha_{25}(a) & 0 \\
\alpha_{32}(a) & \cdots & \alpha_{35}(a) & 0 \\
\alpha_{12}(b) & \cdots & \alpha_{15}(b) & \alpha_{46}(b) \\
\alpha_{22}(b) & \cdots & \alpha_{25}(b) & \alpha_{56}(b) \\
\alpha_{32}(b) & \cdots & \alpha_{35}(b) & 0
\end{array}\right|,
$$

and

$$
D_{n}^{[21]}=\left|\begin{array}{cccc}
\alpha_{12}(a) & \cdots & \alpha_{15}(a) & 0 \\
\alpha_{32}(a) & \cdots & \alpha_{35}(a) & 0 \\
\alpha_{12}(b) & \cdots & \alpha_{15}(b) & \alpha_{46}(b) \\
\alpha_{22}(b) & \cdots & \alpha_{25}(b) & \alpha_{56}(b) \\
\alpha_{32}(b) & \cdots & \alpha_{35}(b) & 0
\end{array}\right| .
$$

Substituting Eqs. (B3) and (B4) into the expression of the structural impedance in (B1) yields (after few manipulations involving the Wronskian)

$$
z_{s n}(a, \phi)=i \frac{\rho_{1} c_{s}^{2}}{\omega a} \frac{D_{n}^{[21]}}{D_{n}^{[11]}} .
$$

\section{APPENDIX C: PROOF REAL PART OF STRUCTURAL IMPEDANCE MATRIX IS ZERO FOR LOSSLESS SYSTEMS}

Assume a closed three-dimensional body with no internal losses and a pressure field on its surface $\Omega$ driving the body into vibration. This pressure field $p(\mathbf{x}), \mathbf{x} \in \Omega$ might arise from the scattering of an incident wave off the surface. The structural impedance matrix $\mathbf{Z}_{s}$ is represented as above but in functional form by $Z_{s}(\mathbf{x}, \mathbf{y})$ where $\mathbf{y} \in \Omega$. The normal velocity response of the surface is $v(\mathbf{y})$. The time average power $\Pi$ injected into the structure - the time average work done by these forces due to the motion of the surface is given by

$$
\Pi=\frac{1}{2} \Re\left[\int_{\Omega} p(\mathbf{x}) v(\mathbf{x})^{*} d^{2} \mathbf{x}\right] .
$$

When there are no losses within the structure, the work done and power injected into the structure must be zero so that there is no buildup or loss of energy over time in the interior space. Thus $\Pi$ is zero. The definition of the structural impedance matrix Eq. (3) in continuous form is

$$
p(\mathbf{x})=-\int_{\Omega_{c}} Z_{s}(\mathbf{x}, \mathbf{y}) v(\mathbf{y}) d^{2} \mathbf{y} .
$$

Inserting this into Eq. (C1) yields

$$
\Pi=-\frac{1}{2} \Re\left[\int_{\Omega_{c}} \int_{\Omega} Z_{s}(\mathbf{x}, \mathbf{y}) v(\mathbf{y}) v(\mathbf{x})^{*} d^{2} \mathbf{x} d^{2} \mathbf{y}\right]=0 .
$$

Due to reciprocity, the impedance matrix must be symmetric, $Z_{s}(\mathbf{x}, \mathbf{y})=Z_{s}(\mathbf{y}, \mathbf{x})$, thus

$$
\Pi=-\frac{1}{2} \Re\left[\int_{\Omega_{c}} \int_{\Omega} Z_{s}(\mathbf{y}, \mathbf{x}) v(\mathbf{y}) v(\mathbf{x})^{*} d^{2} \mathbf{x} d^{2} \mathbf{y}\right]=0 .
$$

Interchanging the dummy variables and adding the result to Eq. (C2) leads to

$$
\begin{aligned}
\Pi & =-\frac{1}{2} \Re\left[\int_{\Omega_{c}} \int_{\Omega} Z_{s}(\mathbf{x}, \mathbf{y})\left(v(\mathbf{x}) v(\mathbf{y})^{*}+v(\mathbf{x})^{*} v(\mathbf{y})\right) d^{2} \mathbf{x} d^{2} \mathbf{y}\right] \\
& =-\Re \int_{\Omega_{c}} \int_{\Omega} Z_{S}(\mathbf{x}, \mathbf{y}) \Re\left[v(\mathbf{x}) v(\mathbf{y})^{*}\right] d^{2} \mathbf{x} d^{2} \mathbf{y}=0 .
\end{aligned}
$$

Because $v(\cdot)$ is an arbitrary function Eq. (C3) can vanish only if $\Re\left[Z_{s}(\mathbf{x}, \mathbf{y})\right]=0$ for all points on the surface, and we conclude that the real part of the structural impedance must vanish.

${ }^{1}$ Yu. I. Bobrovnitskii, "Impedance theory of sound scattering: General relations," Acoust. Phys. 52(5), 513-517 (2006).

${ }^{2}$ E. G. Williams, Fourier Acoustics: Sound Radiation and Nearfield Acoustical Holography (Academic, London, 1999), Chap. 8, pp. 251-293. ${ }^{3} \mathrm{C}$. Langrenne, M. Melon, and A. Garcia, "Boundary element method for the acoustic characterization of a machine in bounded noisy environment," J. Acoust. Soc. Am. 121, 2750-2757 (2007).

${ }^{4}$ I. Lucifredi and H. Schmidt, "Subcritical scattering from buried elastic shells," J. Acoust. Soc. Am. 120(6), 3566-3583 (2006).

${ }^{5}$ A. T. Abawi and M. B. Porter, "Propagation in an elastic wedge using the virtual source technique," J. Acoust. Soc. Am. 121(3), 1374-1382 (2007).

${ }^{6}$ N. P. Valdivia and E. G. Williams, "Study of the comparison of the methods of equivalent sources and boundary element methods for near-field acoustic holography," J. Acoust. Soc. Am. 120, 3694-3705 (2006).

${ }^{7}$ M. Zampolli, F. B. Jensen, and A. Tesei, "Benchmark problems for acoustic scattering from elastic objects in the free field and near the seafloor," J. Acoust. Soc. Am. 125, 89-98 (2009).

${ }^{8}$ C. F. Gaumond and T. Yoder, "Determination of structural impedance from scattering data," J. Acoust. Soc. Am. 93(3), 1415-1422 (1995).

${ }^{9} \mathrm{G}$. V. Borgiotti, "Effect of the compliance on the scattering of an elastic object immersed in fluid: A general formulation," J. Acoust. Soc. Am. 87(3), 1055-1061 (1990).

${ }^{10}$ Yu. I. Bobrovnitskii, "Impedance theory of sound scattering and absorption: A constrained best absorber and the efficiency bounds of passive scatterers and absorbers," Acoust. Phys. 53(1), 113-118 (2007).

${ }^{11}$ Yu. I. Bobrovnitskii, K. D. Morozov, and T. M. Tomilina, "A periodic surface structure with extreme acoustic properties," Acoust. Phys. 56(2), 127-131 (2010).

${ }^{12}$ S. E. S. Karlsson, "Identification of external structural loads from measured harmonic responses," J. Sound Vib. 196(1), 59-74 (1996).

${ }^{13}$ Yu. I. Bobrovnitskii, "The problem of vibration field reconstruction: Statement and general properties," J. Sound Vib. 247(1), 145-163 (2001).

${ }^{14}$ M. C. Junger and D. Feit, Sound, Structures, and Their Interaction, 2nd ed. (Acoustical Society of America, New York, 1993), Chap. 1, pp. 1-14.

${ }^{15}$ L. Brennan, J. Mallet, and I. Reed, "Rapid convergence rate of adaptive arrays," IEEE Trans. Aerosp. Electron. Syst. 10(6), 853-863 (1974).

${ }^{16}$ R. L. Weaver and O. I. Lobkis, "Diffuse fields in open system and the emergence of the Green's function (1)," J. Acoust. Soc. Am. 116(5), 2731-2734 (2004).

${ }^{17} \mathrm{M}$. Campillo and A. Paul, "Long-range correlations in the diffuse seismic coda," Science 299, 547-549 (2003). 
${ }^{18}$ P. Roux, W. A. Kuperman, and NPAL group, "Group extracting coherent wave fronts from acoustic ambient noise in the ocean," J. Acoust. Soc. Am. 116, 1995-2003 (2004).

${ }^{19}$ K. G. Sabra, P. Gerstoft, P. Roux, W. A. Kuperman, and M. C. Fehler, "Surface wave tomography from microseisms in southern California," Geophys. Res. Lett. 32, L14311, doi:10.1029/2005GL023155 (2005).

${ }^{20} \mathrm{O}$. A. Godin, "Emergence of the acoustic Green's function from thermal noise," J. Acoust. Soc. Am. 121, 96-102 (2007).

${ }^{21}$ R. L. Weaver, "Ward identities and the retrieval of Green's functions in the correlations of a diffuse field," Wave Motion 45, 596-604 (2008).

${ }^{22}$ R. Snieder, E. Slob, and K. Wapenaar, "Lagrangian Green's function extraction, with applications to potential fields, diffusion and acoustic waves," New J. Phys. 12, 063013 (2010)

${ }^{23} \mathrm{~F}$. Mulargia and S. Castellaro, "Passive imaging in nondiffuse acoustic wavefields," Phys. Rev. Lett. 100, 218501 (2008).

${ }^{24} \mathrm{~F}$. Mulargia and S. Castellaro, "Nondiffuse elastic and anelastic passive imaging," J. Acoust. Soc. Am. 127, 1391-1396 (2010).

${ }^{25} \mathrm{Yu}$. I. Bobrovnitskii, "A new impedance-based approach to analysis and control of sound scattering," J. Sound Vib. 297, 743-760 (2006).

${ }^{26}$ R. D. Doolittle and H. Überall, "Sound scattering by elastic cylindrical shells," J. Acoust. Soc. Am. 39, 272-275 (1966).
${ }^{27}$ W. Kuperman and F. Ingenito, "Spatial correlation of surface generated noise in a stratified ocean," J. Acoust. Soc. Am. 67, 1988-1996 (1985).

${ }^{28}$ N. C. Makris, F. Ingenito, and W. A. Kuperman, "Detection of a submerged object insonified by surface noise in an ocean waveguide," J. Acoust. Soc. Am. 96, 1703-1724 (1994).

${ }^{29}$ G. H. Koopmann, L. Song, and J. Fahnline, "A method for computing acoustic fields based on the principle of wave superposition," J. Acoust. Soc. Am. 86, 2433-2438 (1989).

${ }^{30} \mathrm{Yu}$. I. Bobrovnitskii and T. M. Tomilina, "General properties and fundamental errors of the method of equivalent sources," Acoust. Phys. 41, 649-660 (1995).

${ }^{31}$ N. P. Valdivia, E. G. Williams, P. C. Herdic, and B. Houston, "Surface decomposition method for near-field acoustic holography," J. Acoust. Soc. Am. 132, 186-196 (2012).

${ }^{32} \mathrm{G}$. V. Borgiotti, "The power radiated by a vibrating body in an acoustic fluid and its determination from boundary measurements," J. Acoust. Soc. Am. 88(4), 1884-1893 (1990).

${ }^{33}$ J. L. Rousselot, La Diffusion Acoustique Par Des Cibles áElastiques de Forme Géométrique Simple: Théories Et Expériences (Acoustic Scattering of Elastic Object of Simple Geometric Shape: Theory and Experiment) (Centre de Documentation de L'Armement, Paris, 1987), Chap. 12, pp. 334-351. 